\title{
LA GANANCIA DE PESO MATERNO EN LA SEGUNDA MITAD DEL EMBARAZO INFLUYE MAS EN LA MACROSOMIA FETAL INDEPENDIENTEMENTE DEL PESO PREGESTACIONAL
}

\author{
Oscar Antonio Limay Ríos ${ }^{1}$ \& Antonio Mambret Luna Figueroa ${ }^{2}$
}

\begin{abstract}
RESUMEN
Objetivo. Determinar la relación entre la obesidad al inicio, en el segundo y tercer trimestre del embarazo y los recién nacidos macrosómicos atendidos en el Instituto Nacional Materno Perinatal durante el periodo 2003 - 2005. Materiales y métodos: estudio analítico, prospectivo, cohortes. La primera cohorte estuvo conformada por 210 gestantes con adecuado peso pregestacional o sobrepeso y la segunda por 211 gestantes con obesidad materna pregestacional. Se procedió al análisis descriptivo, a través de porcentajes, promedios y desviaciones estándar. Para la determinación de las pruebas de hipótesis se procedió a estadísticas inferenciales significativos con un nivel de significación estadística alfa menor a 0,05 . Para la determinación de la influencia se procedió a la utilización del Riesgo Relativo (RR) con sus intervalos de confianza al $95 \%$. Resultados. La obesidad al inicio, en el segundo y tercer trimestre del embarazo se encontró fuertemente relacionada con los recién nacidos macrosómicos, así mismo la talla materna y la ganancia de peso en el $2^{\circ}$ y $3^{\circ}$ trimestre. Conclusión. La ganancia de peso en el tercer y segundo trimestre durante la gestación influye más en el peso del recién nacido independientemente del peso pregestacional.
\end{abstract}

Palabras clave: Obesidad; Macrosomía; Ganancia de peso;Recién nacido (Fuente: DeCS BIREME).

\section{WEIGHT GAIN MATERNAL IN THE SECOND HALF OF PREGNANCY INFLUENCING THE FETAL MACROSOMIA PREGESTATIONAL REGARDLESS OF WEIGHT}

\begin{abstract}
Objectives. To determine the relationship between obesity at the beginning, in the second and third trimester of pregnancy and macrosomic newborns treated at the National Maternal Perinatal Institute during the period from 2003 to 2005. Materials and methods. An analytical, prospective study in which it was followed two cohorts of pregnant women, the first one with 210 pregnant with adequate prepregnancy weight or overweight; and the second one with 211 pregnant with prepregnancy maternal obesity. We proceeded to descriptive analysis, through percentages, means and standard deviations. For the determination of hypothesis tests proceeded to significant inferential statistics with an alpha level of statistical significance of less than 0.05 . To determine the influence, it proceeded to use the Relative Risk (RR) with confidence intervals at $95 \%$. Results. Obesity at the beginning, in the second and third trimester of pregnancy was found strongly related to macrosomic newborns and same maternal height and weight gain in the $2 \mathrm{nd}$ and $3 \mathrm{rd}$ quarter. Conclusion. Weight gain in the third and second quarters during pregnancy strongest impact on birth weight regardless of pre-pregnancy weight.
\end{abstract}

Key words: Obesity; Macrosomia; weight gain; Newborn (Source:MeSH NLM).

\section{INTRODUCCIÓN}

A nivel global la mortalidad en lactantes y niños menores de cinco años ha disminuido', sin embargo, la salud del neonato en nuestra región no muestra mejoría a pesar de que la cifra de mortalidad infantil ha disminuido y la brecha con los países desarrollados se ha acortado². Existen dos factores que condicionan la mortalidad del neonato: el bajo peso al nacer y la macrosomía neonatal; en los países en vías de desarrollo como el nuestro el bajo peso al nacer es el principal factor determinante conocido de la mortalidad infantil, sin embargo, la macrosomía neonatal presenta una tendencia amenazante a convertirse en el mediano plazo en el principal protagonista de la mortalidad y morbilidad infantil y tener secuelas a largo plazo en la edad adulta de enfermedades relacionadas con el sobrepeso tales como la hipertensión, la diabetes, coronariopatias e infarto, entidades que actualmente están causando millones de muertes en el mundo.

En múltiples estudios hay una correlación del peso materno pregestacional con el peso ganado del bebe al nacer y su repercusión en el futuro en desarrollar obesidad, diabetes o coronariopatías ${ }^{3,4}$, la ganancia de peso en el embarazo, además de estar relacionada con el consumo de alimentos, también tiene que ver con factores ambientales y los de naturaleza psicosocial. Uno de los aspectos relevantes del control prenatal es el diagnóstico, evaluación y manejo de la condición nutricional de la embarazada. Es conocido el hecho de que el peso materno pregestacional y la ganancia ponderal durante el embarazo influyen directamente sobre el peso fetal. Es

Médico GínecoObstetra. Magíster en Salud Pública. Jefe de la Unidad de Investigación. Asistente del Servicio de Medicina Fetal del Instituto Nacional Materno Perinatal. Lima Perú.

2 Médico GínecoObstetra. Profesor Principal de la Universidad Naiconal Mayor de San Marcos. Director Ejecutivo de Investigación, Docencia y Atención en Obstetricia y Ginecología - Instituto Nacional Materno Perinatal - Lima - Perú. 
así como el bajo peso y la obesidad materna se relaciona con recién nacidos pequeños y grandes para la edad gestacional, respectivamente. Alrededor del $8.2 \%$ de los recién nacidos pesan mas de $4000 \mathrm{~g}^{5}$ coincidiendo con las cifras del INMP el año 2002 y para los recién nacidos de bajo peso para la edad gestacional es de un $10 \%{ }^{6}$, así por ejemplo hay trabajos en la que demuestran que hay una correlación entre la restricción del crecimiento intrauterino y la inadecuada nutrición materna ${ }^{7}$.

Los pesos al nacer han aumentado con el tiempo es así que en el INMP para el año 2002 ya habían nacido 1280 neonatos grandes para la edad gestacional ${ }^{6}$. La definición clásica de un recién nacido GEG es de aquel con un peso mayor o igual al percentil 90 para una edad gestacional dada. Este umbral de la anormalidad se denomina normalidad aislada. El uso de tal definición incluye un porcentaje significativo de la población normal, que inevitablemente reduce la potencia de los estudios de investigación para mostrar cambios. El porcentaje de recién nacidos GEG con factores de origen genético, puede superar el $70 \%$. El restante $30 \%$ de lactantes es de los que pueden considerarse anormalmente GEG y requieren atención clínica especial ${ }^{8}$.

El crecimiento y desarrollo del feto son regulados por numerosos factores que incluyen el ambiente uterino materno, el funcionamiento de la placenta y la disponibilidad de nutrientes para la madre y el feto. Hay una fuerte relación entre el peso al nacer y la edad gestacional en embarazos normales. Un neonato que se encuentra por arriba del percentil 90 o mas de más de 4,000 gramos al nacer puede ser cronológica y funcionalmente normal, en tanto que en embarazos complicados por diabetes un recién nacido de las mismas dimensiones puede ser anormalmente grande y tener consecuencias concomitantes, estos lactantes grandes se han relacionado con tasas altas de morbilidad y mortalidad materna y perinatal. La morbilidad de un lactante anormalmente grande puede se producto de traumatismos tanto de la madre como del feto así como de complicaciones metabólicas y respiratorias de este último. La mortalidad perinatal relacionada con un feto grande es mayor que la de los de tamaño normal ${ }^{9}$.

Una serie de procesos que suceden simultáneamente durante el crecimiento del feto, como la diferenciación orgánica, el aumento de peso y el desarrollo de la función corporal, lo prepara para vivir fuera del útero en condiciones óptimas. Los procesos celulares característicos del crecimiento fetal (aumento de masa, síntesis de proteínas y diferenciación) no son bien conocidos. Sin embargo, el genotipo fetal contribuye con casi $15 \%$ de las variaciones en el peso al nacer debido a ciertas tendencias heredadas como género, características raciales - étnicas y contribuciones genéticas maternas y paternas. Casi $2 \%$ de las variaciones en el peso al nacer son atribuibles a los cromosomas sexuales ${ }^{10}$. El feto masculino pretérmino es, en promedio 150 a 200 gramos mayor que el del femenino. Este crecimiento puede deberse al afecto de hormonas testiculares o por una diferencia antigénica más notoria entre el feto masculino y su madre. La placentas de los recién nacidos masculinos también pesan más que las de los femeninos ( $2 \%$ más a las 40 semanas $)^{8}$.

Hay una influencia materna importante en el tamaño fetal y se ha calculado su contribución como de casi $20 \%$. Se ha demostrado que la talla materna tiene relación con el peso al nacer,8; por el contrario, la talla del padre no parece contribuir mucho al tamaño del recién nacido.

La diferencia en el tamaño fetal se hace aparente en el tercer trimestre de la gestación y no antes ${ }^{10}$. En etapas tempranas predominan los factores genéticos. Se puede considerar al crecimiento en etapas tardías de la gestación como resultado de la interrelación entre su causa genética y las influencias de restricción que lo inhiben. Los factores exógenos son más importantes en etapas posteriores de la gestación, cuando se hace evidentes las variaciones del tamaño al nacer. El equilibrio entre influencias genéticas y exógenas (nutrición materna, factores placentarios) es controlado por las hormonas fetales ${ }^{11}$.

A mayor número de embarazos las mujeres tienen pesos cada vez mayores en los embarazos sucesivos. Además, hay una gran probabilidad de que las madres que tienen un hijo grande para la edad gestacional (GEG) o pequeño para su edad gestacional (PEG) tengan uno similar en el siguiente embarazo Existen ciertos factores hormonales que contribuyen con el crecimiento fetal como la Insulina, factores de crecimiento similares a la insulina, tiroxina y la leptina entre otros ${ }^{8}$

Existe extensa bibliografía relacionada al uso del IMC como un indicador para evaluar riesgo de Bajo Peso al Nacer. EI IMC surge de la fórmula Peso/Talla ${ }^{2}$ y clasifica como bajo peso (IMC menor de 19,8), peso normal (IMC entre 19,8 a 26), sobrepeso (IMC entre 26,1 a 28,9) y obesidad (IMC mayor de 29) $)^{12,13}$.

La búsqueda de "estándares de oro" para medir el crecimiento intrauterino es un grave problema, ya que la evaluación de los procedimientos diagnósticos para conocer alteraciones en exceso o en defecto del crecimiento fetal, se realiza recurriendo a un patrón que es muy cuestionado ${ }^{14}$, existen varios trabajos en los cuales de clasifica el peso del recién nacido en función a su edad gestacional, nosotros utilizaremos el valor absoluto de mayor o igual de 4 kilogramos para catalogar a un recién nacido ${ }^{14,15}$, existen otros indicadores antropométricos como: Talla, Perímetro cefálico, Índice de masa corporal, Índice ponderal, Peso al nacer entre circunferencia cefálica, talla entre perímetro cefálico ${ }^{16}$, los cuales no usaremos.

\section{MATERIALES Y MÉTODOS}

Tipo y diseño de Estudio: Prospectivo, Longitudinal, Analítico, Cohortes. Población: Gestantes atendidas en 
el Instituto Nacional Materno Perinatal y controladas, con parto, puerperio y recién nacido atendidos en la institución que cumplan los siguientes criterios de selección. Criterios de inclusión para las expuestas: Obesidad pregestacional $\left(\mathrm{IMC}>30 \mathrm{~kg} / \mathrm{m}^{2}\right)$, Máximo 12 controles prenatales, Feto único, Parto de feto vivo, Feto sin malformaciones, Controlada hasta la fecha del parto en la institución. Criterios de inclusión para las no expuestas: Peso normal o sobrepeso pregestacional (IMC 19.8 a 29.9 $\mathrm{kg} / \mathrm{m}^{2}$ ), Máximo 12 controles prenatales, Feto único, Parto de feto vivo, Feto sin malformaciones, Controlada hasta la fecha del parto en la institución. Criterios de Exclusión para las expuestas: Más de 12 semanas al momento de la captación, Gestante que no recuerden su Fecha de última Menstruación, Diabetes Mellitus manifiesta, Hiperémesis gravídica, Estados de Hipertiroidismo, Estados de hipotiroidismo, Hipertensión inducida por el embarazo, Alguna enfermedad intercurrente del embarazo que condicionen patología materna evidente, Alteración fetal que conlleve a un trastorno del crecimiento del feto, Alteración placentaria que conlleve a un trastorno del crecimiento del feto, Recién nacido pretérmino. Criterios de Exclusión para las no expuestas: Gestante con más de 12 semanas al momento de la captación, No recuerden su Fecha de última Menstruación, Diabetes Mellitus manifiesta, Hiperémesis gravídica, Estados de Hipertiroidismo, Estados de hipotiroidismo, Hipertensión inducida por el embarazo, Alguna enfermedad intercurrente del embarazo que condicionen patología materna evidente, Alteración fetal que conlleve a un trastorno del crecimiento del feto, Alteración placentaria que conlleve a un trastorno del crecimiento del feto, Recién nacido pretérmino. Tiempo: Desde abril del 2003 hasta el 01 de marzo del 2005. Unidad de Análisis: Gestante atendida en el Instituto Nacional Materno Perinatal. Unidad de Muestreo: Historia Clínica Perinatal. Tamaño de la muestra: Cuando el grupo de exposición son: las pacientes con obesidad materna pregestacional y sobrepeso materno pregestacional y el grupo de no exposición son: normal peso materno pregestacional y bajo peso materno pregestacional tenemos la probabilidad de que si las dos muestras difieren esta refleje una verdadera diferencia entre las poblaciones con un nivel de confianza del $95 \%$, con una diferencia significativa o poder del $80 \%$, con una razón de no expuestos y de expuestos de 1:1, con una prevalencia esperada del factor en el grupo no expuestos de $4,9 \%$, con un valor de riesgo relativo esperado del $2,8 \%$ y con una prevalencia del factor en el grupo expuesto de un $13,7 \%$ nuestro tamaño de muestra sale de 386 en total, siendo para el grupo expuesto de 193 y de igual manera en el no expuesto. Tipo de muestreo: No probabilística por conveniencia. Técnica: Observación documental. Instrumento de recolección de datos: Formulario con 32 ítems divididos en dos áreas. La primera corresponde a datos maternos que consta de 27 ítems con respuestas cerradas y abiertas. La segunda área corresponde a datos neonatales con cinco ítems de respuestas abiertas y cerradas. Plan de Tabulación y análisis: Para la adecuada interpretación de los datos se procedió a confeccionar tablas univariadas, divariadas y multivariadas, también se procedió a la confección de gráficos. Plan de Análisis: Para el análisis se procedió a la utilización de estadísticas descriptivas promedios y desviaciones estándar para las variables cuantitativas y porcentajes para las variables cualitativas, para el análisis de ganancia de peso e Índice de Masa Corporal se procedió de la siguiente manera. El Índice de Masa Corporal fue definido como peso en kilogramos dividido por la talla en metros cuadrados $\left(\mathrm{kg} / \mathrm{m}^{2}\right)$ de las gestantes el cual fue clasificado de acuerdo a las recomendaciones de la Organización Mundial de la Salud (OMS): Normal IMC de 19.8 a $24.9 \mathrm{~kg} / \mathrm{m}^{2}$, Sobrepeso 25.0 a $29.9 \mathrm{~kg} /$ $\mathrm{m}^{2}$ y Obesidad mayor a $30 \mathrm{~kg} / \mathrm{m}^{2}$. Los trimestres fueron definidos como primero hasta 14 semanas completas, segundo de 15 a 28 y tercero 29 semanas al parto. El peso estimado ganado por trimestre ${ }^{(33)}$ fue calculado usando la ganancia semanal de peso (diferencia entre el primero y el último peso registrado de ese trimestre, dividido entre el número de semanas que separan las dos observaciones) y multiplicado por el número de semanas correspondientes al trimestre en base a la siguiente formula:

$$
\left(\frac{b-a}{c}\right) d
$$

$a=$ Primer peso del trimestre,$b=$ Ultimo peso del trimestre, $c=$ Diferencia de las semanas que corresponden al peso $a$ y $b, d=$ Numero de Semanas por trimestre correspondiente (14 para el Primer y Segundo Trimestre, y 12 para el Tercer Trimestre)

El total del peso ganado estimado fue hallado mediante la diferencia del último peso registrado menos el peso pregestacional más la ganancia semanal por la diferencia de la edad gestacional al parto según neonatología, menos la edad gestacional del último control. La ganancia de peso fue comparado con las recomendaciones dadas por el Instituto de Medicina (IOM) en 1990 de acuerdo al Índice de Masa Corporal Inicial (Anexo 1). Los pesos estimados ganados por trimestre fueron comparados con la tabla anterior como menor del ideal, ideal y mayor del ideal. Para el análisis de regresión múltiple se considero una ganancia semanal de peso promedio (Anexo 1). Obteniéndose la diferencia del peso por trimestre según el índice de masa corporal inicial según la IOM. Para las inferencias estadísticas se procedió a utilizar el estadístico Chi cuadrado para las variables cualitativas. Para las variables cuantitativas se procedió a utilizar el estadístico Coeficiente de Regresión Múltiple $\left(\mathrm{r}^{2}\right)$ con un nivel de significación estadística Alfa $<0,05$. Para la determinación de la influencia entre la ganancia de peso y el resultado macrosómico de recién nacido se procedió a utilizar el Riesgo Relativo (RR) crudo y ajustado con sus intervalos de confianza al $95 \%$. Técnica de medición de la madre: Las pacientes una vez captadas se centralizaron en un consultorio asignado por el INMP para su seguimiento durante todo el periodo de la gestación. Las mediciones antropométricas de la madre se efectuaron con técnica estandarizada con tallímetro metálico y balanza digital. Se 
pesó a la gestante con la menor cantidad de ropa posible (sólo se quedó con su prenda íntima y su bata delgada), descontándose $300 \mathrm{gr}$ al peso total obtenido; tanto para el peso como para la talla se le medió sin zapatos. Las medidas fueron anotadas en el instrumento de recolección de datos.

El estado nutricional materno se establecerá a través del Índice de masa corporal pregestacional según la OMS, de la ganancia de peso materno según la IOM y del seguimiento se realizará a partir de las 12 semanas. Técnica de medición del recién nacido: Se usó un tallímetro midiendo desde el vertex hasta el talón y para el peso se usará una balanza pediátrica digital. La medición de la antropometría del recién nacido se realizó al nacer. El perímetro cefálico se midió con la cinta métrica pediátrica. Procesamiento de Datos: Programa Estadístico utilizado para efectuar el análisis y procesamiento de los datos. Epi-Info, SPSS, Excel; se realizó los siguientes pasos: clasificación los datos por medio de categorías, revisión crítica de los datos recolectados, establecimiento de un orden o jerarquía entre ellas, análisis y señalamiento de la existencia o inexistencia de relaciones entre los datos, detalle el método analítico a utilizar acorde con el diseño de estudio inicial y presentación de tabla de resultados esperados

\section{RESULTADOS}

Se presentan los resultados en tres grandes áreas: la primera está referida a las características generales de las pacientes, la segunda a las características de los recién nacidos macrosómicos y finalmente la tercera a la relación entre las características de la obesidad materna y la macrosomía al nacimiento.

Tabla 1. Características maternas y obstétricas de las gestantes atendidas en el Instituto Especializado Materno Perinatal 2003-2005

\begin{tabular}{ll}
\hline & X $\pm \mathbf{D S}$ \\
\hline Estatura Materna $(\mathrm{m})$ & $1,5 \pm 0$ \\
\hline Edad Materna (años) & $29 \pm 6$ \\
Peso Pregestacional $(\mathrm{Kg})$ & $63 \pm 0$ \\
\hline IMC Pregestacional $\left(\mathrm{Kg} / \mathrm{m}^{2}\right)$ & $26 \pm 4$ \\
$\quad$ Normal & $23 \pm 1$ \\
Sobrepeso & $27 \pm 1$ \\
Obesidad & $32 \pm 3$ \\
Número de Controles Prenatales & \\
$1^{\circ}$ Trimestre & $2 \pm 1$ \\
$2^{\circ}$ Trimestre & $3 \pm 1$ \\
$3^{\circ}$ Trimestre & $3 \pm 2$ \\
Peso materno $(\mathrm{Kg})$ & \\
$1^{\circ}$ Trimestre & $61 \pm 9$ \\
$2^{\circ}$ Trimestre & $67 \pm 9$ \\
$3^{\circ}$ Trimestre & $72 \pm 9$ \\
\hline IMC materno $\left(\mathrm{Kg} / \mathrm{m}^{2}\right)$ & \\
$1^{\circ}$ Trimestre & $26 \pm 4$ \\
$2^{\circ}$ Trimestre & $29 \pm 3$ \\
$3^{\circ}$ Trimestre & $31 \pm 4$ \\
\hline
\end{tabular}

Tanto la estatura materna, así como la edad corresponden a población joven, presentando todos los estadios de peso materno según Índice de Masa Corporal (IMC) al comenzar el embarazo. El comportamiento de los controles prenatales bordea lo recomendado por el Ministerio de Salud, aunque un poco menor, siendo menos en el primer trimestre y en promedio tres en el tercer trimestre. Al final del embarazo se mantiene todos los estadios de peso materno, según medición en kilogramos y por IMC (Tabla 1).

Al estudiar las variables del recién nacido según la obesidad materna en el tercer trimestre, se encontró que en promedio el peso del recién nacido fue mayor para las obesas, este dato fue estadísticamente significativo. Del mismo modo se evidenció que había mayor talla en el recién nacido de madres obesas. No hubo marcadas diferencias para el IMC del recién nacido, perímetro cefálico, edad gestacional e Índice de Apgar al minuto y a los cinco minutos, este último dato no fue estadísticamente significativo. En cuanto a los resultados del parto por cesárea, se evidenció que esté fue mayor para las obesas $(50,7 \%)$ que para las no obesas $(40,3 \%)$, este dato fue estadísticamente significativo. Al estudiar la variable macrosomía del recién nacido, se encontró que esté fue mayor en las obesas que en las no obesas, este dato fue estadísticamente significativo (Tabla 2).

Los recién nacidos macrosómicos con madres con obesidad previa al embarazo, representaron el $13.25 \%$, existiendo en ellas 1,6 veces más probabilidad de presentar recién nacido macrosómico, que cuando no se presenta obesidad previa al embarazo. (RR: 1.64 IC 95\% 0,85 - 3,16). Del mimo modo al estudiar esta característica en el segundo trimestre del embarazo, se encontró $17,19 \%$ de macrosómicos con madres en obesidad, existiendo tres veces más riesgo de presentar macrosomía cuando la madre presenta obesidad en el segundo trimestre (RR: 3,05 IC 95\% 1,68 - 5,55); comportamiento similar se observó en las que en el tercer

Tabla 2. Características del recién nacido de madres obesas y no obesas en el $3^{\circ}$ trimestre Instituto Nacional Materno Perinatal 2003-2005

\begin{tabular}{lccc}
\hline & $\begin{array}{c}\text { Obesa } \\
\mathbf{n = 2 1 1}\end{array}$ & $\begin{array}{c}\text { No Obesa } \\
\mathbf{n = 2 1 0}\end{array}$ & $\mathbf{p}$ \\
\hline Peso al nacer $(\mathrm{Kg})$ & $3484 \pm 5$ & $3292 \pm 4$ & $<0.001$ \\
\hline Talla $(\mathrm{cm})$ & $50,1 \pm 2$ & $49,7 \pm 2$ & 0.060 \\
IMC del recién nacido $\left(\mathrm{Kg} / \mathrm{m}^{2}\right)$ & $1,4 \pm 0$ & $1,3 \pm 0$ & \\
Perímetro Cefálico $(\mathrm{cm})$ & $35,1 \pm 2$ & $34,6 \pm 1$ & 0.001 \\
Edad Gestacional $(\mathrm{sem})$ & $39,3 \pm 1$ & $39,1 \pm 1$ & 0.101 \\
Índice de Apgar & & & \\
$\quad$ 1' & $8,1 \pm 1$ & $8,3 \pm 1$ & 0.031 \\
$\quad$ 5' & $8,9 \pm 1$ & $8,9 \pm 0$ & 0.469 \\
Cesárea (\%) & 50,7 & 40,3 & $<0.001$ \\
Sexo (\%) & & & 0.076 \\
$\quad$ Femenino & 44,1 & 51,9 & \\
$\quad$ Masculino & 55,9 & 48,1 & \\
Macrosómico (\%) & 13,7 & 4,3 & $<0.001$ \\
Bajo Peso al Nacer (\%) & 4,1 & 3,8 & 0.632 \\
\hline
\end{tabular}


trimestre en que presentaron obesidad materna, el 13,7\% de las gestantes, existiendo 3,2 veces más riesgo de tener bebé macrosómico (RR: 3,21 IC95\% 1,56 - 6,60); Se observa que el riesgo aumenta rápidamente entre el primer y segundo trimestre y muy discretamente del segundo al tercer trimestre (Tabla 3).

Al estudiar la estatura materna y su relación con la macrosomía se observa que, a mayor estatura, mayor el riesgo de presentarlo, siendo de 1,03 cuando se encuentra entre $1,56 \mathrm{~m}$ a $1,65 \mathrm{~m}$ a 8 cuando esta es mayor a 1,66 metros. Al estudiar el comportamiento del Peso (Ideal o no ideal) según la ganancia de peso al segundo trimestre, encontramos que solamente fue significativo para macrosomía, cuando la ganancia de peso materno al segundo trimestre es mayor al ideal, existiendo 8,7 veces más riesgo de presentar macrosomía (RR: 8,7 IC95\% 2,1 - 35, 8). Del mismo modo se evidencia que cuando la ganancia de peso materno total es mayor a lo ideal, mayor es el riesgo de presentar macrosomía, al compararlo con los que presentan una ganancia de peso normal (Tabla 3).

Tabla 3. Riesgo relativo de Macrosomía según variables maternas y obstétricas Instituto Especializado Materno Perinatal 2003-2005

\begin{tabular}{|c|c|c|c|c|c|}
\hline & \multirow{2}{*}{$\mathbf{N}$} & \multicolumn{2}{|c|}{ Macrosómico } & \multirow{2}{*}{$\mathbf{R R}$} & \multirow{2}{*}{ IC (95\%) } \\
\hline & & $\mathrm{n}$ & $\%$ & & \\
\hline $\begin{array}{l}\text { Obesidad Previa al } \\
\text { Embarazo }\left(\mathrm{Kg} / \mathrm{m}^{2}\right)\end{array}$ & & & & 1,6 & $(0,9-3,2)$ \\
\hline$I M C \geq 30$ & 83 & 11 & 13,3 & & \\
\hline $\mathrm{IMC}<30$ & 347 & 28 & 8,1 & & \\
\hline $\begin{array}{l}\text { Obesidad } 2^{\circ} \text { Trimestre } \\
\left(\mathrm{Kg} / \mathrm{m}^{2}\right)\end{array}$ & & & & 3,1 & $(1,7-5,6)$ \\
\hline IMC $\geq 30$ & 128 & 22 & 17,2 & & \\
\hline $\mathrm{IMC}<30$ & 302 & 17 & 5,6 & & \\
\hline $\begin{array}{l}\text { Obesidad } 3^{\circ} \text { Trimestre } \\
\left(\mathrm{Kg} / \mathrm{m}^{2}\right)\end{array}$ & & & & 3,2 & $(1,6-6,6)$ \\
\hline IMC $\geq 30$ & 219 & 30 & 13,7 & & \\
\hline $\mathrm{IMC}<30$ & 211 & 9 & 4,3 & & \\
\hline \multicolumn{6}{|l|}{ Edad Materna (años) } \\
\hline$\leq 19$ & 35 & - & - & & \\
\hline $20-24$ & 84 & 3 & 3,6 & & \\
\hline $25-29$ & 128 & 9 & 7,0 & $1,8^{*}$ & $(0,5-6,7)$ \\
\hline $30-34$ & 112 & 19 & 17.0 & $1,8^{*}$ & $(0,8-4,2)$ \\
\hline$>34$ & 71 & 8 & 11,3 & $0,6^{*}$ & $(0,1-2,8)$ \\
\hline \multicolumn{6}{|l|}{ Estatura maternal $(\mathrm{m})$} \\
\hline$\leq 1,45$ & 41 & 3 & 7,3 & - & \\
\hline $1,46-1,6$ & 251 & 19 & 7,6 & $0,9^{*}$ & $(0,8-1,0)$ \\
\hline $1,56-1,7$ & 129 & 15 & 11,6 & $1,0^{*}$ & $(0,9-1,2)$ \\
\hline$>1,7$ & 9 & 2 & 22,2 & 8,0 & $(1,3-50)$ \\
\hline \multicolumn{6}{|l|}{ Trimestre } \\
\hline$<$ Ideal & 139 & 9 & 6,5 & 3,5 & $(0,8-15,7)$ \\
\hline Ideal & 107 & 2 & 1,9 & 1,0 & \\
\hline $\begin{array}{l}>\text { Ideal } \\
\end{array}$ & 166 & 27 & 16,3 & 8,7 & $(2,1-35,8)$ \\
\hline \multicolumn{6}{|l|}{ Trimestre } \\
\hline$<$ Ideal & 103 & 5 & 4,9 & 0,7 & $(0,3-2,2)$ \\
\hline Ideal & 120 & 8 & 6,7 & 1,0 & \\
\hline$>$ Ideal & 186 & 23 & 12,4 & 1,9 & $(0,9-4,0)$ \\
\hline \multicolumn{6}{|l|}{ Ganancia de Peso Total } \\
\hline$<$ Ideal & 154 & 8 & 5,2 & 2,7 & $(0,6-12,2)$ \\
\hline Ideal & 102 & 2 & 2.0 & 1,0 & \\
\hline$>$ Ideal & 154 & 26 & 16,9 & 8,6 & $(2,1-35,5)$ \\
\hline
\end{tabular}

* RR ajustado a obesidad materna previo al embarazo
La incidencia de macrosómicos aumenta conforme aumenta el IMC pregestacional, siendo un $3,1 \%$ de Macrosomía para los de IMC normal, a 13,25\% para las que presentaron obesidad pregestacional; este comportamiento se evidencia también en las que fueron catalogadas según su IMC, en el segundo trimestre, la incidencia es de $5 \%$ de macrosómicos para los de peso normal, a un $17,5 \%$ para los de obesidad, estos datos fueron estadísticamente significativos. La incidencia de macrosomía guardó una discreta relación con la edad materna, no encontrándose en las adolescentes (<19 años) y aumentando según la edad hasta los 34 años, este dato fue estadísticamente significativo, así mismo, este comportamiento se observó con la talla materna, siendo mayor la incidencia conforme aumentaba la talla (Tabla 4).

Al estudiar la incidencia de macrosomía en relación a la ganancia de peso por trimestres, encontramos que este también va en aumento conforme la ganancia es

Tabla 4. Incidencia de macrosomía según características maternas Instituto Especializado Materno Perinatal 2003 $-2005$

\begin{tabular}{|c|c|c|c|c|c|c|}
\hline \multirow{2}{*}{ Variables } & \multirow{2}{*}{ medida } & \multirow{2}{*}{$\mathbf{N}$} & \multicolumn{2}{|c|}{ Macrosómico } & \multirow{2}{*}{$X^{2}$} & \multirow[b]{2}{*}{$\mathbf{p}$} \\
\hline & & & $\mathrm{n}$ & $\%$ & & \\
\hline IMC Pregestacional & $\mathrm{Kg} / \mathrm{m}^{2}$ & & & & 8.9 & 0.012 \\
\hline Peso normal & & 133 & 4 & 3.1 & & \\
\hline Sobre peso & & 214 & 24 & 11.2 & & \\
\hline Obesidad & & 83 & 11 & 13.6 & & \\
\hline IMC 2T & $\mathrm{Kg} / \mathrm{m}^{2}$ & & & & 15.7 & 0.002 \\
\hline Peso normal & & 60 & 3 & 5 & & \\
\hline Sobre peso & & 224 & 13 & 5.8 & & \\
\hline Obesidad & & 126 & 22 & 17.5 & & \\
\hline Datos perdidos & & 20 & 1 & 5 & & \\
\hline IMC 3T & $\mathrm{Kg} / \mathrm{m}^{2}$ & & & & 14.7 & 0.002 \\
\hline Peso normal & & 16 & 1 & 6.6 & & \\
\hline Sobre peso & & 185 & 6 & 3.2 & & \\
\hline Obesidad & & 228 & 32 & 14.0 & & \\
\hline Datos perdidos & & 1 & - & - & & \\
\hline Edad Materna & años & & & & 16.1 & 0.003 \\
\hline$\leq 19$ & & 35 & - & - & & \\
\hline $20-24$ & & 84 & 3 & 3.6 & & \\
\hline $25-29$ & & 128 & 9 & 7.0 & & \\
\hline $30-34$ & & 112 & 19 & 17.0 & & \\
\hline$>34$ & & 71 & 8 & 11.3 & & \\
\hline Estatura maternal & metros & & & & 3.8 & 0.290 \\
\hline$\leq 1,45$ & & 41 & 3 & 7.3 & & \\
\hline $1,46-1,55$ & & 251 & 19 & 7.6 & & \\
\hline $1,56-1,65$ & & 129 & 15 & 11.6 & & \\
\hline$>1,66$ & & 9 & 2 & 22.2 & & \\
\hline Ganancia de Peso 2T & $\mathrm{Kg}$ & & & & 18.0 & $<0.001$ \\
\hline$<$ Ideal & & 139 & 9 & 6.5 & & \\
\hline Ideal & & 107 & 2 & 1.9 & & \\
\hline$>$ Ideal & & 166 & 27 & 16.3 & & \\
\hline Ganancia de Peso 3T & $\mathrm{Kg}$ & & & & 5.6 & 0.60 \\
\hline$<$ Ideal & & 103 & 5 & 4.9 & & \\
\hline Ideal & & 120 & 8 & 6.7 & & \\
\hline$>$ Ideal & & 186 & 23 & 12.4 & & \\
\hline $\begin{array}{l}\text { Ganancia de Peso } \\
\text { Total }\end{array}$ & $\mathrm{Kg}$ & & & & 21.0 & $<0.001$ \\
\hline$<$ Ideal & & 154 & 8 & 5.2 & & \\
\hline Ideal & & 102 & 2 & 2.0 & & \\
\hline$>$ Ideal & & 154 & 26 & 16.9 & & \\
\hline
\end{tabular}

1T: Primer trimestre, 2T: Segundo Trimestre, 3T: Tercer trimestre. IMC: Índice de Masa Corporal. Kg: Kilogramos 
IMC Pregestacional

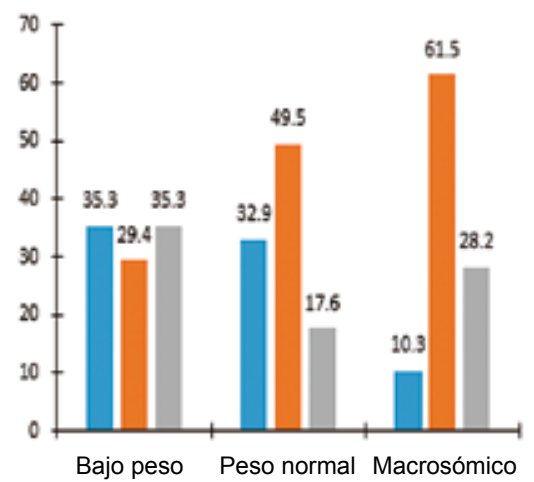

IMC 2do trimestre

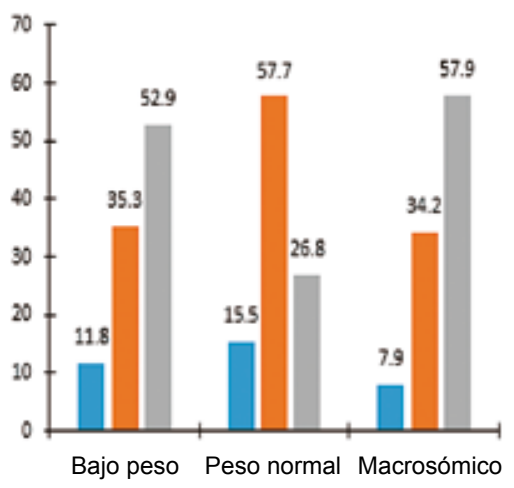

IMC 3er trimestre

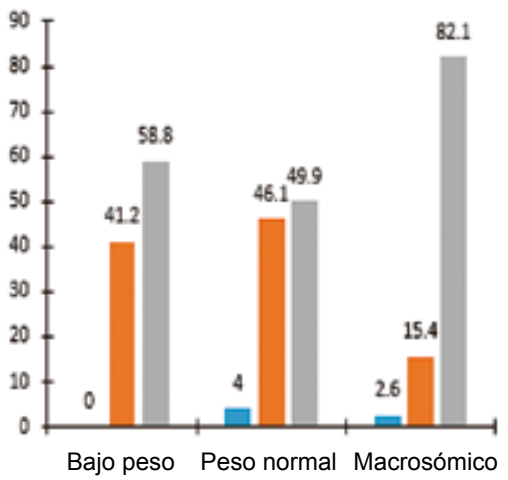

Figura 1. Peso del recién nacido según el Índice de Masa Corporal pregestacional, en el $2^{\circ}$ Trimestre, en el $3^{\circ}$ trimestre Instituto Especializado Materno Perinatal 2003-2005

mayor del ideal en cada trimestre, siendo de $16,3 \%$ en el segundo trimestre y de $12,4 \%$ en el tercer trimestre, este último dato no fue estadísticamente significativo. Al estudiar la ganancia de peso total y la incidencia de macrosomía, se encontrón que este era mayo cuando la ganancia se encontraba por encima de lo ideal, este dato fue estadísticamente significativo (Tabla 4).

Al estudiar la distribución del peso del recién nacido según el IMC pregestacional, encontramos que fue mayor la distribución de macrosómicos para las catalogadas como sobre peso y obesidad que para los del IMC normal. Este comportamiento fue similar al estudiar la distribución del peso del recién nacido con el IMC materno al segundo trimestre, se encontró mayor distribución de macrosómicos en las de IMC con sobrepeso y obesidad que en las normales al igual que la distribución del peso del recién nacido según el IMC al tercer trimestre, encontramos que fue mucho mayor la distribución de macrosómicos para las catalogadas como obesidad que para los del IMC normal (Figura 1).

Al estudiar la relación del IMC pregestacional según las recomendaciones de la IOM y el peso ganado durante el embarazo, encontramos que este se evidencia marcadamente en aumento conforme el peso ganado aumenta, siendo de $6,2 \%$ en la obesas con peso ganado normal, $42,3 \%$ para las de ganancia baja y de 51,5 para las de ganancia excesiva (Tabla 5).

Tabla 5. Peso materno ganado basado en el Índice de Masa Corporal (IMC) Instituto Nacional Materno Perinatal 2003 - 2005

\begin{tabular}{lccccccc}
\hline \multirow{2}{*}{$\begin{array}{l}\text { IMC } \\
\text { pregestacional } \\
\text { según IOM }\end{array}$} & \multicolumn{9}{c}{ Peso ganado } & \multirow{2}{*}{ Normal } & \multicolumn{3}{c}{ Bajo } & \multicolumn{3}{c}{ Excesivo } & X \pm DS \\
\cline { 2 - 6 } & $\mathbf{n}$ & $\%$ & $\mathbf{n}$ & $\%$ & $\mathbf{n}$ & $\%$ & \\
\hline Peso normal & 57 & 30,5 & 87 & 46,5 & 43 & 23,0 & $12,7 \pm 5,0$ \\
Sobrepeso & 39 & 31,0 & 26 & 20,6 & 61 & 48,4 & $11,2 \pm 5,4$ \\
Obesidad & 6 & 6,2 & 41 & 42,3 & 50 & 51,5 & $7,1 \pm 6,3$ \\
Total & 102 & 24,9 & 154 & 37,6 & 154 & 37,6 & $10,9 \pm 5,9$ \\
\hline
\end{tabular}

Al estudiar la relación entre las variables edad materna, estatura materna, IMC pregestacional, al $2^{\circ}$ y $3^{\circ}$ trimestre y su diferencia de peso ganado según IOM encontramos significancias estadísticas que nos permitieron determinar que los eventos son relacionados a la macrosomía del recién nacido (Tabla 6).

Tabla 6. Modelo de regresión lineal múltiple para el peso e Recién Nacido Instituto Especializado Materno Perinatal 2003-2005

\begin{tabular}{|c|c|c|c|c|}
\hline \multirow[t]{2}{*}{ Variables } & \multicolumn{3}{|c|}{$\begin{array}{l}\text { Coeficiente de } \\
\text { regresión }\end{array}$} & \\
\hline & B & ES & $\mathbf{p}$ & \\
\hline Estatura Materna & 1513 & 430 & $<0.001$ & 1 \\
\hline IMC $2^{\circ}$ Trimestre & -156 & 36 & $<0.001$ & 1 \\
\hline $\begin{array}{l}\text { Diferencia de peso ganado } 2^{\circ} \\
\text { Trimestre }\end{array}$ & 32 & 9 & 0.001 & 2 \\
\hline $\begin{array}{l}\text { Diferencia de peso ganado } 3^{\circ} \\
\text { Trimestre }\end{array}$ & -55 & 18 & 0.002 & 3 \\
\hline IMC $3^{\circ}$ Trimestre & 88 & 30 & 0.004 & 4 \\
\hline IMC Pregestacional & 78 & 29 & 0.008 & 5 \\
\hline $\begin{array}{l}\text { Diferencia de peso ganado al final } \\
\text { del embarazo }\end{array}$ & 39 & 16 & 0.015 & 6 \\
\hline Edad Materna & 7 & 4 & 0.095 & 7 \\
\hline
\end{tabular}

$R^{2}=0.168$

\section{DISCUSIÓN}

En el embarazo la obesidad es una situación de alto riesgo. Actualmente, el aumento de las tasas de obesidad es parte de una epidemia mundial que tiene mayor prevalencia en los países desarrollados. No obstante, este problema afecta a más de la mitad de las mujeres peruanas, según la OMS en el 2000 el $46,8 \%$ y de las mujeres se encuentran en riesgo de obesidad y el $13.1 \%$ son obesas ${ }^{17}$. Desde hace varias décadas muchos estudios han reportado numerosas complicaciones obstétricas y perinatales debidos a la obesidad materna ${ }^{18-23}$.

El indicador más sensitivo de composición corporal y de obesidad es el IMC, que proporciona información sobre 
el nivel de reservas energéticas y sobre los componentes de la masa corporal total ${ }^{24}$. Los hallazgos encontrados señalan que el riesgo de macrosomía se ve incrementado con el aumento del IMC de la madre, según los reportes, las mujeres obesas con IMC > 30 tienen 2 a 3.36 veces más riesgo de tener hijos macrosómicos, igualmente la proporción de macrosómicos en mujeres obesas va desde 8 al $17.2 \%^{19,20,23,25-27}$. Los mecanismos para la causa de macrosomía en madres obesas no está aun bien definida, aunque la macrosomía es más común en madres no diabéticas en comparación con las madres con diabetes gestacional, Lipecq ${ }^{18}$ encontró que el $80 \%$ de los recién nacidos macrosómicos son nacidos de madres diabéticas, está bien aceptado la clásica relación entre hiperglicemia materna como causa de hiperinsulinemia fetal originando la macrosomía. No obstante, el desarrollo de la macrosomía ha sido observado en fetos nacidos de mujeres obesas con normal tolerancia a la glucosa. Esto sugiere que los mecanismos que promueven el excesivo crecimiento fetal son más complejos que la clásica consecuencia de la hiperinsulinemia fetal, y la relación de la obesidad con macrosomía puede reflejar un inherente síndrome de resistencia a la insulina que, al tiempo del test de tolerancia a la glucosa, muestra una compensación metabólica ${ }^{18}$. Los resultados obtenidos en el presente estudio confirman que la obesidad aumenta el riesgo de macrosomía, este riesgo se ve incrementado en caso de obesidad en el segundo y tercer trimestre del embarazo.

Así como está documentado la relación del IMC materno con el peso del recién nacido, también se ha estudiado la asociación entre la ganancia excesiva de peso materno con la macrosomía ${ }^{27}$. El excesivo incremento de peso aumenta el riesgo de macrosomía, Jensen D. encontró que en gestantes que ganaban más de $15 \mathrm{~kg}$ durante el embarazo eran cuatro veces más probables de tener recién nacidos macrosómicos en comparación de las que ganaba de 5 a $10 \mathrm{Kg}^{(22,27)}$. Estudios previos han demostrado que la ganancia de peso gestacional dentro de los rangos recomendados por el Instituto de Medicina (IOM) es asociado con mejor resultado para madres e infantes. De acuerdo a las recomendaciones del Instituto de Medicina, el excesivo incremento de peso es un factor asociado a la macrosomía ${ }^{28}$; asimismo, la obesidad durante el embarazo y la ganancia excesiva de peso están asociados a un aumento de morbilidad de la madre y del feto, incluyendo retención de peso materno postparto y adicionalmente, el exceso de peso del recién nacido parece estar asociado a un posterior exceso de peso en años posteriores de la vida ${ }^{29}$. En la presente investigación se demostró el efecto del excesivo peso ganado como un factor de riesgo asociado a la macrosomía en especial al segundo trimestre, así como al peso total ganado durante el embarazo. La ganancia de peso esta asociado significativamente al ingreso energético a través del término del segundo trimestre de embarazo y, por el consumo de energía controlado. Del mismo modo Johnsten y Kandel ha descrito una relación entre la ganancia de peso en el segundo y tercer trimestre con el peso al nacer, encontrando que las tasas más altas de ganancia de peso fueron asociadas con recién nacidos grandes para la edad gestacional y menores tasas de ganancia de peso con recién nacidos de menor tamaño ${ }^{30}$. En el presente estudio, se encontró un mayor riesgo para recién nacidos macrosómicos en gestantes que tuvieron exceso de ganancia de peso en el segundo trimestre que en el tercer trimestre.

Por otro lado existen diversos estudios que indican que un gran número de mujeres embarazadas tienen una ganancia de peso sobre el rango recomendado por la $\mathrm{IOM}^{22,27,29,31}$. Baeter encontró que alrededor del $60 \%$ de mujeres obesas y con sobrepeso pregestacional tenían un excesivo incremento de peso y sólo el $26 \%$ estaba dentro de lo recomendado. En otros estudios este exceso de ganancia de peso va entre 24 a $38 \%{ }^{29,31}$. Asimismo existe una tendencia de una ganancia excesiva de peso a medida que incrementa el IMC materno provocando resultados perinatales adversos. En nuestra población más del $50 \%$ de las mujeres obesas tenían una excesiva ganancia de peso.

Cabe mencionar que a pesar de que un gran número de recién nacidos macrosómicos se explica por un IMC materno alto tanto previo al embarazo como durante al embarazo, también existen otros factores que determinarían un feto macrosómico, como parte de un componente del desarrollo. Esto implica que la temporal tendencia en el incremento de peso al nacer y macrosomía no es enteramente debido al incremento en el IMC materno y obesidad materna aunque parece ser la principal ${ }^{18}$, pero, también puede estar determinado por el peso al nacer de la madre que podría predecir el peso del recién nacido ${ }^{25}$, del mismo modo, Caughey et al encontró que la ganancia de peso previo al embarazo aumenta el riesgo de resultados adversos inclusive en mujeres que no están en sobrepeso incluyendo un mayor riesgo de grandes para la edad gestacional. La ganancia de 1 a 2 unidades del IMC en un promedio de 2 años aumenta el riesgo de GEG en mujeres que no necesariamente necesitan llegar a sobrepeso $u$ obesidad para aumentar el riesgo ${ }^{32,33}$.

Por otro lado, si vemos el promedio de peso ganado durante el embarazo, Winkvist et al encontró que para mujeres indonésicas ${ }^{34}$ fue $8.3 \pm 3.6$, y Abrams encontró un promedio de $11.2 \pm 4.1$ para gestantes iraníes con sobrepeso al inicio del embarazo ${ }^{35}$, y Grados encontró en una población peruana un aumento de $7.4 \pm 4.2$ en mujeres obesas, y $10.6 \pm 3,9$ en mujeres con peso normal ${ }^{36}$, similares a lo hallado en este estudio.

Los hallazgos encontrados muestran que existe una asociación con el recién nacido macrosómico que se incrementa con la edad materna $22,25,27,28$. El hecho de que el incremento de IMC está asociado con incremento de la edad materna ${ }^{19,21}$ puede reflejar la tendencia para la 
población ganar peso con la edad. Por otro lado se ha reportado que las mujeres tienden a ganar peso con cada embarazo, es decir incrementa su IMC según su paridad, por consiguiente mayores tasas de fetos macrosómicos. En este estudio el riesgo de macrosomía no fue significativo probablemente porque a mayor edad también se incremento el riesgo de recién nacidos con bajo peso.

Con respecto a la estatura materna y el riesgo de recién nacido macrosómicos Leary en el 2006 encontró que la talla materna es generalmente un fuerte predictor de tamaño fetal, así como la circunferencia cefálica materna de la circunferencia fetal, a comparación de los efectos de la talla e IMC paterno fueron más débiles que a su equivalente en la madre ${ }^{37}$. En el presente estudio se halló un riesgo incrementado de acuerdo a la talla materna en especial mayor de $1,65 \mathrm{~m}$ resultados que son consistentes con otros estudios ${ }^{25}$.

En cuanto al sexo del recién nacido, tradicionalmente la literatura reporta dentro de los riesgos de macrosomía es sexo masculino, Cuttié y Rodrigues han encontrado un mayor porcentaje de recién nacidos macrosómicos masculinos $62.5 \%$ y $64 \%$ respectivamente ${ }^{27,38}$, aunque Savona-Ventura y Callaway no encontraron diferencias significativas en de sexo. En nuestros hallazgos el sexo de los recién nacido macrosómicos fue significativamente para los de sexo masculino, probablemente debido a que los fetos masculinos tienden a ganar mayor peso que el femenino.

Por otro lado, una ganancia excesiva de peso y un mayor IMC aumenta la tasa de cesárea ${ }^{23,39}$. Sebire encontró que el aumento de la tasa de cesárea en mujeres embarazadas obesas fue sobre el $20 \%$ comparado con un aproximado de $10 \%$ para el grupo de peso normal ${ }^{32}$. El aumento de cesáreas puede ser consecuencia de un aumento de la tasa macrosómicos que conducen a una desproporción durante el trabajo de parto o posiblemente a que la contractibilidad uterina es menor a lo óptimo en un subgrupo de mujeres obesas, además el incremento de depósito de grasas en tejidos blandos de la pelvis, incluyendo el aumento de riesgo de hemorragia posparto por el aumento de sangrado en GEG por haber una mayor área de implantación de la placenta ${ }^{23}$. Las tasas cesáreas en mujeres obesas encontradas por algunas investigaciones varían de 32 a $39 \%$ a comparación de 16.6 al $27,1 \%$ en mujeres con peso normal previo al embarazo ${ }^{20-22,35}$. Jensen ${ }^{22}$ encontró un riesgo de 3.6 veces de cesáreas en gestantes con ganancia de peso mayor de $15 \mathrm{Kg}$. Lo hallado en el presente estudio encuentra un mayor porcentaje de cesáreas en mujeres obesas durante al final del embarazo en comparación de las mujeres no obesas, de la misma forma los recién nacidos macrosómicos tuvieron mayores tasas de cesáreas que los recién nacidos de peso normal; aunque la tasa de cesáreas mostradas en ambos grupos es considerablemente mayor a lo registrado en otros países.
Cabe mencionar que inversamente a lo encontrado del mayor riesgo de macrosomía en mujeres obesas o con un exceso en el aumento de peso, se evidenció que la proporción de recién nacidos con bajo peso en mujeres obesas también fue alto, esto conlleva a interpretar estos resultados según la propuesta de Allen de Finlandia quien encontró que las gestantes con sobrepeso y obesas frecuentemente consumen una menor cantidad de micro nutrientes en su dieta, y por consiguiente un mayor riesgo de anemia, lo cual conducen a un crecimiento fetal reducido en algunas de ellas ${ }^{40}$.

La limitación de este estudio incide en la confiabilidad de los pesos previos registrados por la gestante que en algunas ocasiones tienden a ser subestimados de su verdadero peso y, según otras investigaciones, el grado de subestimación puede ser más grande para mujeres de alto peso. En el presente estudio, esas subestimaciones podrían afectar en una mala clasificación del riesgo de exposición solamente entre mujeres cuya cantidad de subestimación cause una diferente clasificación a la categoría de IMC o en el peso ganado al final del embarazo.

\section{CONCLUSIONES}

- El peso del recién nacido está directamente relacionado con la obesidad pregestacional y con la ganancia de peso en el segundo y tercer trimestre de gestación.

- La ganancia de peso en el tercer y segundo trimestre durante la gestación influye más en el peso del recién nacido independientemente del peso pregestacional

- El excesivo aumento de peso conlleva a un mayor riesgo de recién nacidos macrosómicos, en especial en el segundo trimestre de gestación, así como también la talla materna.

- Incluir en futuras investigaciones otras variables que pueden ser confusoras como el nivel socioeconómico y nivel educativo.

- Se hace necesario llevar a cabo un trabajo de investigación más amplio en los diferentes medios socioculturales de nuestro país.

\section{REFERENCIAS BIBLIOGRÁFICAS}

1. Washington, DC, 26 de setiembre de 2002 (OPS) - La 26a Conferencia Sanitaria anamericana, que reúne esta semana en Washington a los ministros de Salud de las Américas

2. Belizán JM. Perinatal health in Latin America. In: Carrera L, eds World congress of Perinatal Medicine 5. Barcelona, septamber 2001. Bologna: Monduzzi; 2001. p. 19-24.

3. Langer $\mathrm{O}$. Is normoglycemia the correct threshold to prevent complications in the pregnant diabetic? Diabetes Review 1996;4:2-10

4. United States Bureau of Vital Statistics Vital Statistics for the United States. Vol. 1 Natality. Washington, DC: USBVS, 1966. 
5. United States Bureau of Vital Statistics Vital Statistics for the United States. Vol 1 Natality. Washington, DC:USBVS. 1970.

6. Informe Estadístico Anual de la Oficina de Estadística e Informática del IEMP. Año 2002 Frenk JP, Situación actual de la salud publica en México, revista de salud pública, México. Año 2000

7. Frenk JP, Situación actual de la salud publica en México, revista de salud pública, México. Año 2000

8. Polani PE.Chromosomal and other genetic influences on firth weight variation. In: Elliot K, Knight J, eds; Size at Birth. Amsterdam: Associated Scientific Publisher, 1974:127-164.

9. Kloosterman GJ. On intrauterina growth: The significance of prenatal care. J.Gynecol Obstet. 1970;8:895.

10. Ounsted M. Ounsted $C$. On fetal growth rate: Its variations and their consequences. In: Clinics in Development Medicine (46). Spastics International Medical Publications. Philadelphia, PA: Lippincott, 1974.

11. Fujikura T, Froehlich IA. Birthwieght, gestational age, and renal glomerural de velopment as indices of fetal maturity $\mathrm{Am}$ J Obstet Gynecol. 1972; 113:627-631.

12. Rosso P. Mardones S. Et al. Curva Patrón de incrementos ponderales para la embarazadas chilenas. "Ministerio de Salud de Chile. 1986

13. Atalah S.E. y cols. Clasificación del estado nutricional de la embarazadas según Indice de Masa corporal y edad gestacional. Centro de Nutrición Humana. Facultad de Medicina Universidad de Chile. Chile 1997

14. Fescina R.H., y Schwarcz R. Crecimiento intrauterino. La mujer gestante. En Crecimiento y Desarrollo, editores Cuminsky M., Moreno E.M., y Suárez E.N., OPS, Publicación Científica N510. 1988, Washington D.C. pag. 71-89. 20

15. OMS. Prevención de la mortalidad y morbilidad perinatales. Ser Inf Téc 457, Ginebra, 1970. 27

16. Jaso L. Neonatología Práctica 1995 Editorial el manual moderno pag 86-90

17. World Health Organization. (Sitio en Internet). Hallado en: http:// http://www.who.int/bmi/index.jsp. Acceso 5 de mayo de 2007.

18. Savona-Ventura C, Gatt M. Short-term obstetrics outcomes in obese Maltese women. Int J Diabetes Metab 2006; 14: 88-91

19. Missouri Department of Health \& Senior Services Center for Health Information Management \& Evaluation. Maternal Obesity and Pregnancy Outcomes. October 2004.

20. Baeten JM, Bukusi EA, Lambe M. Pregnancy complications and outcomes among overweight and obese nulliparous women. Am J Public Health. 2001; 91:436-40.

21. Callaway L, Prins J, Chang A, Mclintyre $H$. The prevalence and impact of overweight and obesity in an Australian population. MJA 2006; 184: 56-9.

22. Jensen DM, Ovesen $P$, Beck-Nielsen $H$, Molsted-Pedersen L, Sorensen B, Vinter C, et al. gestational weight gain and pregnancy outcomes in 481 obese glucose-tolerant women. Diabetes Care 2005; 28:2118-22.

23. Sebire NJ, Jolly M, Harris JP, Wadsworth J, Joffe M, Beard RW, et al. Maternal obesity and pregnancy outcome: a study of 287213 pregnancies in London. Int J Obesity 2001; 25: 1175-82.

24. Sánchez A, Del Real SI, Solano L, Peña E, Adela M. Índice de masa corporal al comienzo del embarazo en un grupo de gestantes venezolanas de bajo estrato socioeconómico y su relación con la antropometría de sus recién nacidos. ALAN 2006; 56 (2): 141-5.

25. Surkan PJ, Hsieh C, Johanson AL, Dickman P, Cnattingius $\mathrm{S}$. Reasons for increasing trends in large for gestational age births. Obstet Gynecol 2004; 104: 720-6.

26. Weiss JL, Malone FD, Emig D, Ball RH, Nyberg DA, Comstock $\mathrm{CH}$, et al. Obesity, obstetric complications and cesarean delivery rate-A population-based screening study. Am J Obstet Ginecol2004; 190:1091-7.

27. Rodrigues T, Teles TP, Barros H. Risk factors for macrosomía in infants of nondiabetic women. Arq Medic 1999; 13 (supl 5): 20-3.

28. Kac G, Velasquez-Melendez G. Gestational weight gain and macrosomía in a cohort of mothers and their children. J Pediatr (Rio J). 2005 Jan-Feb;81(1):47-53.

29. Bertoldi L, Bartholow B, Serrate S, Branchtein L, Schmidt M, Fleck T. Assessment of

30. Suitor CW. Maternal weight: a report of an expert work group. National Center for Education in Maternal and Child Health 1997.

31. Rhodes JC, Schoendorf KC, Parker JD. Contribution of excess weight gain during pregnancy and macrosomía to the cesarean delivery rate, 1990-2000. Pediatrics 2003;111:1181-5.

32. Caughey AB. Obesity, weight loss, and pregnancy outcomes. The Lancet 2006; 368 (9542).

33. Villamor E, Cnattingius $S$. Interpregnancy weight change and risk of adverse pregnancy outcomes: a population-based study. The Lancet 2006; 368 (9542) 1164-7.

34. Winkvist A, Stenlund H, Hakimi M, Nurdiati DS, Dibley MJ. Weight-gain patterns from pregnancy until delivery among women in Central Java, Indonesia. Am J Clin Nutr 2002; 75:1072-7.

35. Abrams B, Altman SL, Pickett KE. Pregnancy weight gain: still controversial. Am J Clin Nutr 2000; 71 (suppl):1233S-41S.

36. Grados F, Cabrera R, Diaz J. Estado nutricional pregestacional y ganancia de peso materno durante la gestación y su relación con el peso del recién nacido. Rev Med Hered 2003; 14: 128-33.

37. Leary S, Fall C, Osmond C, Lovel H, Campbell D, Eriksson J, et al.Geographical variation in relationships between parental body size and offspring phenotype at birth. Acta Obstet Gynecol Scand. 2006;85(9):1066-79.

38. Cutié ML, Figueroa M, Segura A, Lestayo C. Macrosomía fetal. Su comportamiento en el último quinquenio. Rev Cubana Obstet Ginecol 2002; 28(1): 34-41

39. Stotland NE, Hopkins LM, Caughey AB. Gestational weight gain, macrosomía and risk of cesarean birth in nondiabetic nulliparas. Obstet Gynecol 2004; 104: 671-7

40. Mardones F. Evolución de la antropometría maternal y del peso de nacimiento en Chile, 1987-2000. Rev Chil Nutr. 2003; 30 (2): 122-31. 


\section{Anexo 1}

\begin{tabular}{lcc}
\hline $\begin{array}{c}\text { Índice de Masa } \\
\text { Corporal Inicial }\end{array}$ & $\begin{array}{c}\text { Ganancia de peso al } \\
\text { primer trimestre }\end{array}$ & $\begin{array}{c}\text { Peso ganado al } \\
\text { final del embarazo }\end{array}$ \\
\hline Bajo peso $<19.8$ & $2.3 \mathrm{Kg}$ & $12.5-18$ \\
Normal $19.8-26$ & $1.8 \mathrm{Kg}$ & $11.5-16$ \\
Sobrepeso $26.1-29$ & $1.3 \mathrm{~kg}$ & $7-11.5$ \\
\hline Obesidad $>29$ & $0.9 \mathrm{Kg}$ & $6-7$ \\
\hline
\end{tabular}

Del cual se desprende el siguiente cuadro, considerando que la ganancia de peso en el segundo y tercer trimestre es constante.

\begin{tabular}{lcc}
\hline $\begin{array}{c}\text { Índice de Masa } \\
\text { Corporal Inicial }\end{array}$ & $\begin{array}{c}\text { Ganancia de peso } \\
\text { en el Segundo } \\
\text { Trimestre }\end{array}$ & $\begin{array}{c}\text { Ganancia de peso } \\
\text { en el tercer trimestre }\end{array}$ \\
\hline Normal & $5.2-7.6$ & $4.4-7.6$ \\
Sobrepeso & $3.1-5.5$ & $2.7-4.8$ \\
Obesidad & $2.7-3.3$ & $2.4-2.8$ \\
\hline
\end{tabular}

Los pesos estimados ganados por trimestre fueron comparados con la tabla anterior como menor del ideal, ideal y mayor del ideal.

Para el análisis de regresión múltiple se considero una ganancia semanal de peso promedio:

\begin{tabular}{lcccc}
\hline $\begin{array}{c}\text { Índice de } \\
\text { Masa Corporal } \\
\text { Inicial }\end{array}$ & $\begin{array}{c}\text { Ganancia } \\
\text { de peso } \\
\text { semanal }\end{array}$ & $\begin{array}{c}\text { Ganancia en } \\
\text { el Segundo } \\
\text { Trimestre }\end{array}$ & $\begin{array}{c}\text { Ganancia } \\
\text { en el Tercer } \\
\text { Trimestre }\end{array}$ & $\begin{array}{c}\text { Ganancia } \\
\text { al final del } \\
\text { Embarazo }\end{array}$ \\
\hline Normal & 450 & 6.3 & 5.4 & 13.75 \\
Sobrepeso & 300 & 4.2 & 3.6 & 9.25 \\
Obesidad & 200 & 2.8 & 2.4 & 6.5 \\
\hline
\end{tabular}

Obteniéndose la diferencia del peso por trimestre según el índice de masa corporal inicial según el Instituto nacional de los Estados Unidos (IOM)
ORCID iDs

Antonio Limay Rios

Antonio Luna Figueroa https://orcid.org/0000-0001-6012-3705

https://orcid.org/0000-0003-1527-8649 